\title{
Is "Cholinergic" Stimulus Useful for Ulcerative Colitis Treatment?
}

\author{
Yu-Chen Pai ${ }^{1} \cdot$ Linda Chia-Hui Yu ${ }^{1}$
}

Published online: 7 November 2019

(c) Springer Science+Business Media, LLC, part of Springer Nature 2019

Pyridostigmine, an acetylcholinesterase inhibitor that increases acetylcholine levels in neuromuscular junctions and postganglionic parasympathetic nerve-targeted organ junctions, is a drug previously used for protection of the military from nerve agents and is routinely used clinically for the treatment of myasthenia gravis. Inhibition of the enzyme acetylcholinesterase impairs the hydrolysis and removal of acetylcholine in order to facilitate repolarization in the synapse. Enteric nerves in the submucosal and myenteric plexus of the gut wall are categorized in the postganglionic parasympathetic division and also release acetylcholine in order to regulate intestinal functions. Based on the long-term observation that smokers (with stimulation of nicotinic acetylcholine receptors) seldom develop T helper (Th) 2-type ulcerative colitis (UC), whereas the habit of smoking seems to worsen the symptoms of Th1-type Crohn's disease (CD), the effect of pyridostigmine as a potential therapy for UC was investigated in a preclinical study by Singh et al. published in this issue of Digestive Diseases and Sciences [1].

The article reported that pyridostigmine bromide (PB) given by intraperitoneally implanted osmotic minipumps for 2 weeks ameliorated colitis severity in a dextran sodium sulfate (DSS)-induced UC mouse model. A dose of $2 \mathrm{mg} /$ day $/ \mathrm{kg}$ body weight starting 7 days prior to providing $3 \%$ DSS in the drinking water was given to the mice and continued for 8 days during DSS drinking until the day of killing. Reduced body weight loss and histopathological score associated with increased tight junctional expression as measured by ZO-1 staining on colonic tissues were found in the PB group but not in the saline group. Other improved eosinophil-related parameters related to DSS challenge, including peripheral eosinophil counts, eotaxin, and in particular IL-5 and IL-13 levels, were significantly decreased in the PB group compared with the saline group. The data

Linda Chia-Hui Yu

lchyu@ntu.edu.tw

1 Graduate Institute of Physiology, National Taiwan University College of Medicine, Suite 1020, \#1 Jen-Ai Rd. Sec. I,

Taipei 100, Taiwan, ROC indicated that long-term use of PB as a treatment for colitis should be studied since the affected cytokines have effects outside of eosinophil differentiation. IL-5 is also involved in supporting the growth and terminal differentiation of B cells into antibody-secreting cells, and IL-13 is involved in elastin remodeling $[2,3]$. The modulation of IL-5 may impact secretory IgA production in the intestine, which is crucial for maintaining a normal microbiota composition and mucosal anti-microbial function [4-6].

In the article by Singh et al., DSS-induced bacterial dysbiosis, which correlated with colitis severity, was reversed or prevented by the use of PB through an unknown mechanism. It is plausible that IL-5-modulated secretory IgA production may be involved in the changes of microbiota composition. Moreover, the detailed mechanisms of how PB decreased IL-5 and IL-13 production originating from either T lymphocytes or mast cells through the elevation of acetylcholine levels warrant further studies. Whether the increase in acetylcholine levels associated with PB may alter the expression of choline acetyltransferase (ChAT, an enzyme required for acetylcholine synthesis) in neurons and non-neuronal cells such as T lymphocytes [7] by a negative feedback loop would be an another interesting mechanism to uncover.

Increased transport of gut flora into subepithelial tissue is thought to be one of the initiating factors contributing to the pathogenesis of inflammatory bowel disease (IBD), especially UC. Elevated gut paracellular permeability and endotoxemia often precede symptom flare in $\operatorname{IBD}[8,9]$. The study of Singh et al. offered some insight into the contribution of acetylcholine toward the organization of epithelial junctional barrier and microbial composition. Treatment with PB increased epithelial tight junction protein staining that correlated with a restoration of specific bacterial species in the fecal microbial population in the DSS mouse model. Functional tests such as luminal-to-serosal macromolecular flux and tissue conductance should be performed to complement the evaluation of epithelial barrier integrity in addition to measurement of junctional protein expression. The results in Singh et al. were in keeping with previous in vitro studies demonstrating that muscarinic cholinergic stimulation 
increased transepithelial electrical resistance and tight junctional expression in epithelial cell lines $[10,11]$. Nevertheless, activation of muscarinic receptors was also involved in increased transcytotic passage of macromolecules in rat gut tissues in models of psychological stress [12]. The impact of cholinergic stimuli on the divergent regulation of transcellular versus paracellular permeability of intestinal epithelia has yet to be explored in models of UC. Visualization of transepithelial bacterial internalization by electron microscopic techniques and in situ hybridization $[13,14]$ would help distinguish transcellular from paracellular changes upon cholinergic stimulation. Additional studies are warranted in order to characterize the mechanisms of acetylcholineinduced macromolecular transcytosis, in particular, clathrinor caveolin-dependent transcytotic pathways. Importantly, the acetylcholine-mediated increase in transcellular passage of macromolecules and possibly bacteria, either through intestinal epithelia or M cells on Peyer's patches, may be involved in the regulation of mucosal immunity and serve as a driving force for altering microbial composition [15-17].

The report by Singh et al. demonstrated that PB inhibited the DSS-induced loss of Clostridia and Flavobacteria and increased the abundance of Erysipelotrichia and Fusobacteria in the fecal microbiota. The key question is whether microbial dysbiosis is a cause or effect of chronic inflammation. If the changes in microbial composition-gain or loss of particular bacterial strains-may provoke the onset of colitis, then what causes gut dysbiosis and the emergence of commensal-derived pathobionts in the first place? Further evaluation of previously reported mucosa-associated bacterial strains with colitogenic ability [5], such as Escherichia coli, Enterococcus faecalis, and Bacteroides subspecies, would add persuasive information regarding the alteration of microbial composition by cholinergic modulation.

In this study, PB administered prior and during DSS drinking attenuated mucosal inflammation and increased the staining of tight junctional proteins in a UC-like model. It would be interesting to investigate the detailed mechanisms and time courses of the underlying mucosal and microbial alterations conferred by PB. Other recent reports also pointed out the possibility of cholinergic modulation for treatment of IBD by experimental models and clinical trials. A murine study showed that ChAT-positive T lymphocytes strengthened the acute innate immune response following mucosal barrier disruption but supported the later resolution process in DSS-induced colitis [18]. Vagus nerve stimulation alleviated colitis severity and was suggested as an alternative approach to anti-inflammatory drugs [19, 20]. Another aspect to reflect on is that cholinergic stimuli acting as secretagogues promoted colonic ion secretion through activation of both muscarinic and nicotinic receptors on epithelial cells [21]. Cholinergics may facilitate outflux and clearance of the colitogenic mucosa-associated bacteria; however, the use of pro-secretory drugs would also raise concerns for aggravating diarrheal symptoms. In sum, cholinergic medication could be applied in the future for UC management but cautions should be taken regarding the timing and duration of its use which may result in different outcome.

\section{References}

1. Singh SP, Chand H, Banerjee S, Agarwal H, Raizada V, Roy S, Sopori ML. Acetylcholinesterase inhibitor pyridostigmine bromide attenuates gut pathology and bacterial dysbiosis in a murine model of ulcerative colitis. Dig Dis Sci. (Epub ahead of print). https://doi.org/10.1007/s10620-019-05838-6.

2. Takatsu K. Interleukin-5 and IL-5 receptor in health and diseases. Proc Jpn Acad Ser B Phys Biol Sci. 2011;87:463-485.

3. Ingram JL, Slade D, Church TD, et al. Role of matrix metalloproteinases-1 and -2 in interleukin-13-suppressed elastin in airway fibroblasts in asthma. Am J Respir Cell Mol Biol. 2016;54:41-50.

4. Bunker JJ, Erickson SA, Flynn TM, et al. Natural polyreactive IgA antibodies coat the intestinal microbiota. Science. 2017;358:eaan6619.

5. Yu LC. Microbiota dysbiosis and barrier dysfunction in inflammatory bowel disease and colorectal cancers: exploring a common ground hypothesis. J Biomed Sci. 2018;25:79.

6. Huang YJ, Pai YC, Yu LC. Host-microbiota interaction and intestinal epithelial functions under circadian control: implications in colitis and metabolic disorders. Chin J Physiol. 2018;61:325-340.

7. Ramirez VT, Godinez DR, Brust-Mascher I, et al. T-cell derived acetylcholine aids host defenses during enteric bacterial infection with Citrobacter rodentium. PLoS Pathog. 2019;15:e1007719.

8. Rojo OP, Roman ALS, Arbizu EA, et al. Serum lipopolysaccharide-binding protein in endotoxemic patients with inflammatory bowel disease. Inflamm Bowel Dis. 2007;13:269-277.

9. Tibble JA, Sigthorsson G, Bridger S, et al. Surrogate markers of intestinal inflammation are predictive of relapse in patients with inflammatory bowel disease. Gastroenterology. 2000;119:15-22.

10. Uwada J, Yazawa T, Islam MT, et al. Activation of muscarinic receptors prevents TNF-alpha-mediated intestinal epithelial barrier disruption through p38 MAPK. Cell Signal. 2017;35:188-196.

11. Dhawan S, Hiemstra IH, Verseijden C, et al. Cholinergic receptor activation on epithelia protects against cytokine-induced barrier dysfunction. Acta Physiol (Oxf). 2015;213:846-859.

12. Gareau MG, Jury J, Perdue MH. Neonatal maternal separation of rat pups results in abnormal cholinergic regulation of epithelial permeability. Am J Physiol Gastrointest Liver Physiol. 2007;293:G198-G203.

13. Wu LL, Peng WH, Kuo WT, et al. Commensal bacterial endocytosis in epithelial cells is dependent on myosin light chain kinaseactivated brush border fanning by interferon-gamma. Am J Pathol. 2014;184:2260-2274.

14. Yu LC, Shih YA, Wu LL, et al. Enteric dysbiosis promotes antibiotic-resistant bacterial infection: systemic dissemination of resistant and commensal bacteria through epithelial transcytosis. Am J Physiol Gastrointest Liver Physiol. 2014;307:G824-G835.

15. Hollander D, Kaunitz JD. The "leaky gut": tight junctions but loose associations. Dig Dis Sci. (Epub ahead of print). https://doi. org/10.1007/s10620-019-05777-2.

16. Yu LC, Wei SC, Ni YN. Interplay between the gut microbiota and epithelial innate signaling in colitis-associated colon carcinogenesis. Cancer Res Front. 2017;3:1-28. 
17. Yu LC. Commensal bacterial internalization by epithelial cells: an alternative portal for gut leakiness. Tissue Barriers. 2015;3:e1008895.

18. Willemze RA, Brinkman DJ, Welting O, et al. Acetylcholineproducing T-cells augment innate immune driven colitis but are redundant in T-cell driven colitis. Am J Physiol Gastrointest Liver Physiol. 2019;317:G557-G568. https://doi.org/10.1152/ajpgi .00067 .2019 .

19. Ghia JE, Blennerhassett $\mathrm{P}$, Kumar-Ondiveeran H, et al. The vagus nerve: a tonic inhibitory influence associated with inflammatory bowel disease in a murine model. Gastroenterology. 2006;131:1122-1130.
20. Bonaz B, Sinniger V, Hoffmann D, et al. Chronic vagus nerve stimulation in Crohn's disease: a 6-month follow-up pilot study. Neurogastroenterol Motil. 2016;28:948-953.

21. Bader S, Diener M. Novel aspects of cholinergic regulation of colonic ion transport. Pharmacol Res Perspect. 2015;3:e0139.

Publisher's Note Springer Nature remains neutral with regard to jurisdictional claims in published maps and institutional affiliations. 